\title{
The Role of Radiotherapy and Chemotherapy in the Treatment of Primary Adult High Grade Gliomas: Assessment of Patients for These Treatment Approaches and the Common Immediate Side Effects
}

\author{
E. E. Philip-Ephraim, ${ }^{1}$ K. I. Eyong, ${ }^{2}$ U. E. Williams, ${ }^{1}$ and R. P. Ephraim ${ }^{1}$ \\ ${ }^{1}$ Department of Internal Medicine, College of Medical Sciences, University of Calabar, PMB 1278, Nigeria \\ ${ }^{2}$ Department of Paediatrics, College of Medical Sciences, University of Calabar, PMB 1278, Nigeria
}

Correspondence should be addressed to E. E. Philip-Ephraim, nneninge@yahoo.com

Received 11 October 2012; Accepted 6 November 2012

Academic Editors: H. Al-Ali, J. Bentel, N. Fujimoto, and L. Mutti

Copyright ( 2012 E. E. Philip-Ephraim et al. This is an open access article distributed under the Creative Commons Attribution License, which permits unrestricted use, distribution, and reproduction in any medium, provided the original work is properly cited.

Gliomas are the commonest primary brain tumours in adults. They are usually classified and graded according to the criteria by the World Health Organisation. High-grade gliomas are the most malignant primary brain tumours. Conventional therapies include surgery, radiotherapy, and chemotherapy. The tumours often demonstrate high levels of resistance to these conventional therapies, and in spite of treatment advances the prognosis remains poor.

\section{Introduction}

Primary brain tumours are tumours that arise within the brain tissue and the environs while secondary tumours originate from elsewhere in the body and spread to the brain [1]. The main functional cell of the brain is called the neuron, while glial cells make up the supporting structures. These glial cells are of different types, namely, the oligodendrocytes which make myelin, astrocytes which take part in neurotransmission and neuronal metabolism, and the ependyma cells which line the ventricles and central canal of the spinal cord. Tumours arising from glial cells are called gliomas. These tumours are the most frequent primary tumours of the central nervous system $[2,3]$.

The World Health Organisation (WHO) classifies astrocytomas into four grades, depending on the microscopic pattern of the tumour, which include increased cellularity, nuclear atypia, mitosis, and vascular proliferation/necrosis. The different characteristics demonstrate the invasiveness and rate of growth of the various grades.

WHO Grade I (Pilocytic astrocytoma) is that which does not demonstrate any of the microscopic patterns.
WHO Grade II (diffuse astrocytoma) is characterized by only atypia.

WHO Grade III (anaplastic astrocytoma) shows both atypia and mitosis.

WHO Grade IV (glioblastoma multiforme) shows areas of necrosis and/or vascular proliferation $[1,4]$.

The tumours in WHO Grades I and II are referred to as low-grade gliomas while those in grades III and IV are called high-grade gliomas (HGG). By far, the HGG are the commonest primary brain tumours in adults $[5,6]$. They can develop de novo or result from a progression of the lowgrade gliomas [7-9]. Seventy percent of the 22,500 new cases of malignant primary brain tumours diagnosed in adults in the United States are due to HGG exhibiting an incidence of 5 per 100,000/per year $[4,10]$. The most common type of HGG is glioblastoma accounting for $80 \%$ of the malignant gliomas while the Oligodendrogliomas constitute about $20 \%$ of glial tumours [11].

Males are more frequently affected than females with a ratio of $3: 2[3,11]$. A possible protective effect of the female hormones has been proposed as a cause since the difference is more noticeable among women of child-bearing age [3]. 
The HGG can occur in any part of the brain but normally involve the cerebral hemispheres [11]. Glioblastoma affects elderly people with a mean age at diagnosis of 64 years while anaplastic astrocytoma affects younger patients of about 45 years $[4,11]$. The codeletion of $1 p / 19 q$ in anaplastic Oligodendrogliomas correlates with better survival and chemosensitivity. Poor survival in anaplastic astrocytoma is linked to loss of phosphate and tensin homolog (PTEN) tumour suppressor gene. Also poor survival in glioblastoma patients is linked to epidermal growth factor receptor overexpression, p53 expression, and MDM2 overexpression [3]. The HGG are highly aggressive malignant tumours with very poor prognosis. The mean survival for anaplastic astrocytoma is $3-5$ years and much shorter, less than 1 year, for glioblastoma patients [12]. Prognostic features include performance status, age, and histologic characteristics [13].

Headache is the initial symptom in about $30 \%$ of cases. The headaches, generally dull in character, are difficult to differentiate from tension headaches. Symptoms of increased intracranial pressure may be present. Seizures can also occur but are less common in glioblastomas probably due to decreased life expectancy in these patients [14]. Cognitive impairment can occur in up to $40-60 \%$ of patients with glioblastoma. Personality and mood changes do also occur. A focal neurological deficit with weakness of one side of body has been reported $[4,11,14]$.

\section{The Role of Radiotherapy}

The treatment of HGG entails different modalities including surgery followed by radiotherapy and chemotherapy. This multimodality approach is still regarded as the mainstay of treatment $[13,15,16]$. Occasionally, when complete resection is impossible, radiotherapy can then be the initial treatment. A thorough treatment planning is therefore necessary for a favourable outcome in radiotherapy [17]. HGG is noted for local recurrence and because of this studies have led to ways of maximizing radiation doses locally to enhance control.

2.1. Conventional Radiotherapy. Various studies carried out in the 1960's to compare the outcome of those receiving radiotherapy (RT) with nonrecipient reported better prognosis in the group that received RT $[18,19]$. Despite this, debate regarding the best way to deliver RT remains. A delay in commencement of RT after surgery has been associated with poor prognosis [20].

2.1.1. Dose. Several studies have been carried out comparing radiation dose of $45 \mathrm{~Gy} 20$ fractions over 4 weeks to $60 \mathrm{~Gy}$ 30 fractions over 6 weeks as relates to the outcome of HGG. A higher dose of 60 Gy was found to correlate with a better prognosis as relates to overall survival $[10,21]$. In another trial, a higher dose of 70 Gy showed no further improvement in survival compared with those that received 60 Gy dose, proving that a dose higher than $60 \mathrm{~Gy}$ had no extra advantage $[18,19]$ but rather can be associated with increased toxicity [10].
2.1.2. Radiation Volume. The $60 \mathrm{~Gy}$ whole brain RT utilized in the 1960's and 1970's in treatment of HGG is now restricted to patients with multifocal gliomas. Local brain irradiation with margins of $2 \mathrm{~cm}$ around the tumour mass is being preferred [18].

This is because of better tumour localization by CT and MRI in this era and also due to reports of tumour recurrence at the primary site in over $90 \%$ of cases [10]. Local brain irradiation has the beneficial effect of reducing the volume of brain tissue irradiated thereby reducing the toxic effect of the radiation without affecting tumour control [19]. Few studies have investigated the volume of radiotherapy delivery. No statistical difference was found in survival rate and morbidity between the groups that received whole brain RT and local brain RT $[22,23]$ whereas performance status was found to be better in recipients of localized field RT [23].

\subsection{Altered Fractionation}

(i) Hyperfractionated radiotherapy involves the giving of a higher total dose than the conventional irradiation using multiple small-sized fractions over the same overall treatment period. The benefit of this was in allowing a higher dose to be delivered without much morbidity due to lower fraction size. Almost all the studies carried out showed no advantage in the use of hyperfractionated over conventional RT $[18,19,24]$.

(ii) Accelerated hyper-fractionated radiotherapy requires the delivery of multiple fractions in the standard dose over a shortened treatment time. Studies done neither showed increase in toxicity nor improvement in survival rate over the hyperfractionation or the conventional RT [10, 18, 19, 25].

(iii) Hypofractionation, here, larger than normal-sized radiation fractions with a reduced total radiation dose and a shortened overall treatment time is employed. This regimen is used not only in older age but also in patients with poor prognosis and unresectable tumours [19]. Hypofractionation showed glioblastoma patients to have a favourable response in terms of survival rate over the conventional fractionation [26] while other studies concluded that hypofractionation had similar results and tolerance comparable with conventional fractionation $[27,28]$.

2.3. Brachytherapy. This employs the placement of radiation isotopes directly inside and around the tumour. This method significantly increases the delivery of a higher tumour dose radiation without much risk to the surrounding brain tissue. Beneficial effect is seen mostly in patients with good karnofsky score and small unifocal tumours. Studies carried out showed encouraging results in the median survival of these patients $[29,30]$ although other studies could not confirm a statistically survival advantage for brachytherapy [18]. 
2.4. Radiosurgery. This entails the delivery of a stereotactically radiation beam in single fraction to focus on the tumour site.

2.5. Other Modalities. This includes the use of hyperthermia, particle therapy, and radiosensitizers.

\section{The Role of Chemotherapy}

The medical treatment of HGG has been a challenge because the drugs must be able to have not only potent and accurate cytotoxic effect but also be able to penetrate the blood brain barrier (BBB) and overcome any mechanism of drug resistance. Chemotherapy is used concomitantly or as an adjuvant to RT. The inclusion of chemotherapy in HGG is found to improve survival significantly [31].

\subsection{Conventional Chemotherapy}

(i) Nitrosoureas. These are alkylating agents and are lipid-soluble which allows them to overcome the BBB successfully. Examples include carmustine, lomustine, and nimustine. They have been used orally, intra-arterially, and as wafers in the treatment of HGG. Nitrosoureas have been studied in different contexts and found to demonstrate prolongation in survival as relates to extension of tumour progression time [32].

(ii) Procarbazine, lomustine, and vincristine $(P C V)$. In an attempt to improve the efficacy of the nitrosoureas, the combination therapy was utilized. Although beneficial effect of PCV as regards prolongation of both survival and tumour progression time amongst anaplastic glioma patients was established [33], nonetheless no demonstrable difference in survival advantage to PCV over carmustine was found in a retrospective study [34].

(iii) Temozolomide (TMZ). This is a newer alkylating agent with excellent bioavailability. The $\mathrm{O}^{6}$ methylguanine-DNA methyl transferase repair protein $(M G M T)$ has been implicated in the resistance to the alkylating cytotoxic drugs. The outcome of methylation of the MGMT promoter is inactivation of the gene which is said to be responsible for the increased sensitivity to treatment. The benefit of Temozolomide in glioblastoma has been linked to the methylation of the MGMT promoter [35]. TMZ is usually given orally at a dose of $200 \mathrm{mg} / \mathrm{m}^{2}$ daily for 5 days and then repeated after 28 days. Trials with TMZ have been carried out in various contexts. These studies have consistently demonstrated clear benefit creditable to TMZ as regards progression free survival, increase in quality of life, and increase in 2 year survival [36-38]. In one of the studies, glioblastoma patients that were diagnosed newly were given TMZ at a dose of $75 \mathrm{mg} / \mathrm{m}^{2}$ daily for 6 weeks alongside with radiotherapy followed by TMZ alone at a dose of $200 \mathrm{mg} / \mathrm{m}^{2} /$ day for 6 cycles. The total number of patients studied was sixty four. This regimen was well accepted and led to a "median survival time of 16 months, with 1- and 2-year survival rates of $58 \%$ and $31 \%$, respectively" [39]. Because of these outcomes, concurrent use of TMZ and RT is now regarded as the standard treatment for newly diagnosed glioblastoma patients [31].

(iv) Platinum salts. These are also alkylating agents and are used mainly in treating recurrent HGG. Two examples of this class are carboplatin and cisplatin. Carboplatin was shown to be effective against recurrent HGG as $50 \%$ of the assessed patients demonstrated increase in their median survival time of 38 weeks while median time to progression of the tumour was 19 weeks [40].

(v) Topoisomerase 1 and 11 Inhibitors. Etoposide is the prototype of type 11 and exerts its action by inhibiting DNA topoisomerase 11 enzymes [41]. The potent synergistic effect of the platins and etoposide has led to the conduction of various trials. In one of the trials, the combination of carboplatin and etoposide showed a median survival time of 10 months when 30 patients were treated [42]. Examples of type-1 inhibitors include Irinotecan and topotecan. Despite the high toxicity displayed by these drugs, the median survival time of 15 months over the control of 11 months was encouraging when topotecan was used concomitantly in an open-labelled study [43].

(vi) Other Cytotoxics. These include taxane derivatives and anthracyclines. Some investigators found modest beneficial effect in the use of taxane in HGG $[44,45]$.

3.2. Biological Modifiers. These are agents with cytostatic action. Different types have been tried in the treatment of HGG. They include Vitamin A and D derivatives, matrix metalloprotease inhibitors, angiogenesis inhibitors, and signal transduction inhibitors like tamoxifen. Various studies conducted showed successes in some while others failed to prove any benefit as regards progression and survival time when compared with placebos in glioblastoma patients [31].

3.3. Intra-Arterial Chemotherapy. The aim of this mode of therapy is to deliver a sufficient dose of the drug into the tumour site. This regimen has been used in various trials for the management of both recurrent as well as newly diagnosed HGG [46]. Intracarotid and intracerebral studies of cisplatin and carmustine, respectively, as single agents have been encouraging as regards disease stabilization and response rates $[47,48]$. Although intra-arterial administration of drugs has been successful, other drugs like etoposide have produced discouraging outcomes for primary malignant brain tumours [49].

3.4. Autologous Bone Marrow Rescue. In an effort to improve the long-term survival of patients with HGG, the use of highdose chemotherapy followed by autologous bone marrow rescue has been studied in various trials. Even though 
remarkable results were obtained with the use of high dose $\mathrm{BCNU}$, the toxic effects produced were enormous $[50,51]$.

3.5. Polymer-Drug Delivery. This method enhances a sustained local drug delivery and exposure, circumvents the BBB and the unnecessary systemic toxic effects. In a multicentre randomized placebo-controlled trial, the authors were able to demonstrate the effectiveness of carmustine in prolonging survival time in patients with recurrent gliomas [52].

3.6. Intratumoral Chemotherapy. Here treatment is delivered directly into the tumour or tumour bed after resection. Advantages of this mode of therapy include maximal concentration of the drug intratumorally, by-pass of BBB, and reduction in unwanted systemic toxic effects. Success has been reported with this mode of treatment [53].

3.6.1. Blood Brain Barrier. The BBB lowers the efficiency of cytotoxic drugs. In an attempt to disrupt this barrier, intraarterial Mannitol has been used to increase the uptake of the cytotoxic drugs [54].

\section{Assessment of Patients for These Treatment Approaches}

The assessment of patients with HGG requires a multidisciplinary approach involving the neurologists, neurosurgeons, neuropathologists, and the neuropsychologists/neuropsychiatrists.

4.1. The Neurologist Assessment. A complete history of the patient will be taken. A thorough general medical and neurological examination can suggest a diagnosis of gliomas. Disease conditions like hypertension and the Ischaemic heart diseases will have to be excluded. Other comorbidites like asthma, kidney failure, bleeding disorders, diabetes mellitus and hepatitis should be excluded. Investigations should include a full blood count, clotting profile, blood glucose, serum electrolyte, urea, creatinine and grouping, and cross matching [14].

4.2. The Neuropsychologist Assessment. This assessment is vital as the initial symptom can be cognitive impairment which can progress during and even after therapy. Assessment of cognitive function using Karnofski Performance Score is usually carried out. In spite of its limitations, it is still used in the evaluation of quality of life [55].

4.3. The Neuropsychiatry Assessment. Patients with primary brain tumours tend to have depression, apathy, psychosis, and personality changes. Counseling of the patient and usage of different modalities of treatment to stabilize the patient makes the neuropschiatrist indispensable [56].

4.4. The Neuropathologist. The cornerstone for confirmation of HGG is by tissue biopsy. The biopsied sample can then be examined histologically, not only to confirm the diagnosis but also to characterize the different grades of gliomas.
For precision, the pathologic diagnosis is interpreted in relation to the clinical history by the neurologist as well as findings of the neurosurgeons and neuroradiologist [57]. Immunohistochemistry studies can also be done. Thus GFAP (Glial fibrillary acidic protein) seen almost exclusively in HGG can strengthen the diagnosis. Genetic testing that can be done include PTEN, TP53, MDM2, EGFR overexpression, and $1 p / 19 q$ codeletions [58]. These tests have prognostic values in chemotherapy.

4.5. The Neuroradiologist Assessment. Advancement in neuroimaging techniques has significantly improved the assessment of HGG. Magnetic Resonance Imaging (MRI) and Computed Tomography (CT) in addition to playing investigative roles in the diagnosis of HGG are used in planning therapy, and a followup in evaluating the success of the treatment modalities [59]. A nonhomogenous enhancement of the mass is often seen with MRI or CT. Functional MRI can be used in determining the location of speech and motor areas in relation to the tumour prior to surgery [4]. The use of Positron Emission tomography using FDG has also been used in differentiating glioblastoma from anaplastic astrocytoma because of regions of striking hypometabolism signifying necrosis [60]. Single-Photon Emission CT imaging has also been used in detecting recurrent gliomas. Other imaging techniques used include Proton magnetic resonance spectroscopy which distinguishes metabolite levels, contrast enhanced MRI which assesses permeability of blood vessels and perfusion imaging to assess cerebral blood volume [4].

4.6. The Neurosurgeon Assessment. A biopsy of the tumour is usually undertaken by the surgeon. Different methods are used and include needle biopsy, stereotactic biopsy, or free hand biopsy but all are guided by imaging techniques [61].

\subsection{Informed Consent Will Have to Be Given by The Patient}

4.7.1. Common Immediate Side Effects. These are side effects that occur hours to days of commencing therapy.

Radiotherapy. The common immediate side effects here are usually mild and include hair loss, scalp redness, weakness, headache, fever, nausea, and vomiting [62].

Chemotherapy. Most of the cytotoxic drugs cause gastrointestinal effects like nausea, vomiting, loss of appetite, and diarrhea. Headache, dizziness, and fatigue can also occur. Local irritation can occur at IV infusion site of carmustine. Intracarotid carmustine can cause vision loss. Intra-arterial administration of nitrosoureas has also been associated with acute neurotoxicity like seizures and confusion $[46,63]$.

\section{Conclusion}

High-grade glioma remains the commonest primary brain neoplasm with a grave outcome. It has been widely studied in various clinical trials but in spite of the different aggressive approaches to therapy ranging from extensive resective surgery to adjuvant radiotherapy and concomitant/adjuvant 
use of single to combination chemotherapy, the prognosis still remains poor. Recent advances in Neuro-imaging and newer cytotoxics have brought some improvement in survival. A more extensive scientific knowledge might help in unraveling the "mysteries" of HGG. Cure for now is elusive, however all hope is not lost. We look forward to a better tomorrow as the clinical trials of various modalities of treatment continue.

\section{References}

[1] J. Rees, S. Brander, R. Howard et al., "Neuro-oncology," in Neurology, A Queen Square Textbook, C. Clarke, R. Howard, M. Rossor, and S. Shorvon, Eds., pp. 771-822, Blackwell Publishing, Oxford, UK, 2009.

[2] D. Figarella-Branger, C. Colin, B. Coulibaly et al., "Histological and molecular classification of Gliomas," Revue Neurologique, vol. 164, no. 6-7, pp. 505-515, 2008.

[3] N. A. Butowski and S. M. Chang, "Glial tumors: the current state of scientific knowledge," Clinical Neurosurgery, vol. 53, pp. 106-113, 2006.

[4] P. Y. Wen and S. Kesari, "Malignant Gliomas in adults," The New England Journal of Medicine, vol. 359, no. 5, pp. 492-507, 2008.

[5] A. A. Brandes and S. Monfardini, "Treatment of elderly patients with high grade Gliomas," Seminars in Oncology, vol. 30, no. 6, supplement 19, pp. 58-62, 2003.

[6] J. S. Frenel, M. Botti, D. Loussouarn, and M. Campone, "Prognostic and predictive factors for Gliomas in adults," Bulletin du Cancer, vol. 96, no. 4, pp. 357-367, 2009.

[7] A. Perry, "Pathology of low-grade Gliomas: an update of emerging concepts," Neuro-Oncology, vol. 5, no. 3, pp. 168178, 2003.

[8] C. Sarkar, A. Jain, and V. Suri, "Current concepts in the pathology and genetics of Gliomas," Indian Journal of Cancer, vol. 46, no. 2, pp. 108-119, 2009.

[9] P. Kleihues and H. Ohgaki, "Primary and secondary glioblastomas: from concept to clinical diagnosis," Neuro-Oncology, vol. 1, no. 1, pp. 44-51, 1999.

[10] N. Laperriere, L. Zuraw, and G. Cairncross, "Radiotherapy for newly diagnosed malignant glioma in adults: a systematic review," Radiotherapy and Oncology, vol. 64, no. 3, pp. 259273, 2002.

[11] L. M. DeAngelis, "Brain tumors," The New England Journal of Medicine, vol. 344, no. 2, pp. 114-123, 2001.

[12] S. M. Chang, I. F. Parney, W. Huang et al., "Patterns of care for adults with newly diagnosed malignant glioma," Journal of the American Medical Association, vol. 293, no. 5, pp. 557-564, 2005.

[13] F. Laigle-Donadey and M. Sanson, "Pattern of care of highgrade Gliomas,” Revue du Praticien, vol. 56, no. 16, pp. 17791786, 2006.

[14] P. Y. Wen and P. M. Black, "Clinical presentation, evaluation and preoperative preparetion of the patient," in The Gliomas, M. S. Berger and C. B. Wilson, Eds., pp. 328-336, WB Saunders, Philadelphia, Pa, USA, 1999.

[15] D. Croteau and T. Mikkelsen, "Adults with newly diagnosed high-grade Gliomas," Current Treatment Options in Oncology, vol. 2, no. 6, pp. 507-515, 2001.

[16] R. D. Kortmann, B. Jeremic, M. Weller, L. Plasswilm, and M. Bamberg, "Radiochemotherapy of malignant glioma in adults: clinical experiences," Strahlentherapie und Onkologie, vol. 179, no. 4, pp. 219-232, 2003.
[17] D. C. Shrieve, D. F. Deen, and D. A. Larson, "Basic principles of radiobiology and radiotherapy," in The Gliomas, M. S. Berger and C. B. Wilson, Eds., pp. 464-479, WB Saunders, Philadelphia, Pa, USA, 1999.

[18] J. Buatti, T. C. Ryken, M. C. Smith et al., "Radiation therapy of pathologically confirmed newly diagnosed glioblastoma in adults," Journal of Neuro-Oncology, vol. 89, no. 3, pp. 313-337, 2008.

[19] W. J. Curran, C. B. Scott, and S. A. Leibel, "Issues in the use of conventional and altered fractionation radiation therapy for paediatric and adult Gliomas," in The Gliomas, M. S. Berger and C. B. Wilson, Eds., pp. 480-488, WB Saunders, Philadelphia, Pa, USA, 1999.

[20] C. Irwin, M. Hunn, G. Purdie, and D. Hamilton, "Delay in radiotherapy shortens survival in patients with high grade glioma," Journal of Neuro-Oncology, vol. 85, no. 3, pp. 339343, 2007.

[21] N. M. Bleehen and S. P. Stenning, "A Medical Research Council trial of two radiotherapy doses in the treatment of grades 3 and 4 astrocytoma," British Journal of Cancer, vol. 64, no. 4, pp. 769-774, 1991.

[22] M. Kita, T. Okawa, M. Tanaka, and M. Ikeda, "Radiotherapy of malignant glioma-prospective randomized clinical study of whole brain vs local irradiation," Gan No Rinsho, vol. 35, no. 11, pp. 1289-1294, 1989.

[23] R. R. Sharma, D. P. Singh, A. Pathak et al., "Local control of high-grade Gliomas with limited volume irradiation versus whole brain irradiation," Neurology India, vol. 51, no. 4, pp. 512-517, 2003.

[24] M. D. Prados, W. M. Wara, P. K. Sneed et al., "Phase III trial of accelerated hyperfractionation with or without difluromethylornithine (DFMO) versus standard fractionated radiotherapy with or without DFMO for newly diagnosed patients with glioblastoma multiforme," International Journal of Radiation Oncology Biology Physics, vol. 49, no. 1, pp. 71-77, 2001.

[25] H. Keim, P. C. Potthoff, K. Schmidt, M. Schiebusch, A. Neiss, and K. R. Trott, "Survival and quality of life after continuous accelerated radiotherapy of glioblastomas," Radiotherapy and Oncology, vol. 9, no. 1, pp. 21-26, 1987.

[26] B. Glinski, "Postoperative hypofractionated radiotherapy versus conventionally fractionated radiotherapy in malignant Gliomas. A preliminary report on a randomized trial," Journal of Neuro-Oncology, vol. 16, no. 2, pp. 167-172, 1993.

[27] M. Y. Sayin, B. Kaya, B. H. Bakkal, K. Altundag, and M. B. Altundag, "The results of hypofractionated radiotherapy in 31 patients with high-grade Gliomas," Medical Oncology, vol. 24, no. 4, pp. 379-383, 2007.

[28] M. C. C. M. Hulshof, E. C. Schimmel, D. A. Bosch, and D. González González, "Hypofractionation in glioblastoma multiforme," Radiotherapy and Oncology, vol. 54, no. 2, pp. 143-148, 2000.

[29] P. Y. Wen, E. A. Iii, P. M. Black et al., "Long term results of stereotactic brachytherapy used in the initial treatment with glioblastomas," Cancer, vol. 73, no. 12, pp. 3029-3036, 1994.

[30] P. K. Sneed, P. R. Stauffer, M. W. McDermott et al., "Survival benefit of hyperthermia in a prospective randomized trial of brachytherapy boost \pm hyperthermia for glioblastoma multiforme," International Journal of Radiation Oncology Biology Physics, vol. 40, no. 2, pp. 287-295, 1998.

[31] D. Mathieu and D. Fortin, "The role of chemotherapy in the treatment of malignant astrocytomas," Canadian Journal of Neurological Sciences, vol. 33, no. 2, pp. 127-140, 2006. 
[32] M. Huncharek and J. Muscat, "Treatment of recurrent high grade astrocytoma; results of a systematic review of 1415 patients," Anticancer Research, vol. 18, no. 2, pp. 1303-1311, 1998.

[33] V. A. Levin, P. Silver, J. Hannigan et al., "Superiority of post-radiotherapy adjuvant chemotherapy with CCNU, procarbazine, and vincristine (PCV) over BCNU for anaplastic Gliomas: NCOG 6G61 final report," International Journal of Radiation Oncology Biology Physics, vol. 18, no. 2, pp. 321-324, 1990.

[34] M. D. Prados, C. Scott, W. J. Curran, D. F. Nelson, S. Leibel, and S. Kramer, "Procarbazine, lomustine, and vincristine (PCV) chemotherapy for anaplastic astrocytoma: a retrospective review of radiation therapy oncology group protocols comparing survival with carmustine or PCV adjuvant chemotherapy," Journal of Clinical Oncology, vol. 17, no. 11, pp. 3389-3395, 1999.

[35] M. E. Hegi, A. C. Diserens, T. Gorlia et al., "MGMT gene silencing and benefit from temozolomide in glioblastoma," The New England Journal of Medicine, vol. 352, no. 10, pp. $997-$ 1003, 2005.

[36] D. Osoba, M. Brada, W. K. A. Yung, and M. Prados, "Healthrelated quality of life in patients treated with temozolomide versus procarbazine for recurrent glioblastoma multiforme," Journal of Clinical Oncology, vol. 18, no. 7, pp. 1481-1491, 2000.

[37] W. K. A. Yung, R. E. Albright, J. Olson et al., "A phase II study of temozolemide vs. procarbazine in patients with glioblastoma multiforme at first relapse," British Journal of Cancer, vol. 83, no. 5, pp. 588-593, 2000.

[38] R. Stupp, W. P. Mason, M. J. van den Bent et al., "Radiotherapy plus concomitant and adjuvant temozolomide for glioblastoma," The New England Journal of Medicine, vol. 352, no. 10, pp. 987-996, 2005.

[39] R. Stupp, P. Y. Dietrich, S. O. Kraljevic et al., "Promising survival for patients with newly diagnosed glioblastoma multiforme treated with concomitant radiation plus temozolomide followed by adjuvant temozolomide," Journal of Clinical Oncology, vol. 20, no. 5, pp. 1375-1382, 2002.

[40] R. E. Warnick, M. D. Prados, E. E. Mack et al., "A phase II study of intravenous carboplatin for the treatment of recurrent Gliomas," Journal of Neuro-Oncology, vol. 19, no. 1, pp. 69-74, 1994.

[41] K. R. Hande, "Etoposide: four decades of development of a topoisomerase II inhibitor," European Journal of Cancer, vol. 34, no. 10, pp. 1514-1521, 1998.

[42] E. Franceschi, G. Cavallo, L. Scopece et al., "Phase II trial of carboplatin and etoposide for patients with recurrent highgrade glioma," British Journal of Cancer, vol. 91, no. 6, pp. 1038-1044, 2004.

[43] M. W. Gross, R. Altscher, M. Brandtner et al., "Openlabel simultaneous radio-chemotherapy of glioblastoma multiforme with topotecan in adults," Clinical Neurology and Neurosurgery, vol. 107, no. 3, pp. 207-213, 2005.

[44] M. C. Chamberlain and P. A. Kormanik, "Salvage chemotherapy with paclitaxel for recurrent primary brain tumors," Journal of Clinical Oncology, vol. 13, no. 8, pp. 2066-2071, 1995.

[45] M. D. Prados, S. C. Schold, A. M. Spence et al., "Phase II study of paclitaxel in patients with recurrent malignant glioma," Journal of Clinical Oncology, vol. 14, no. 8, pp. 2316-2521, 1996.

[46] E. J. Dropcho, "Intra-arterial chemotherapy for malignant Gliomas," in The Gliomas, M. S. Berger and C. B. Wilson, Eds., pp. 537-547, WB Saunders, Philadelphia, Pa, USA, 1999.
[47] M. S. Mahaley, S. W. Hipp, E. J. Dropcho et al., "Intracarotid cisplatin chemotherapy for recurrent Gliomas," Journal of Neurosurgery, vol. 70, no. 3, pp. 371-378, 1989.

[48] G. B. Bradac, R. Soffietti, A. Riva, G. Stura, S. Sales, and D. Schiffer, "Selective intra-arterial chemotherapy with BCNU in recurrent malignant Gliomas," Neuroradiology, vol. 34, no. 1, pp. 73-76, 1992.

[49] L. G. Feun, Y. Y. Lee, and W. K. A. Yung, "Intracarotid VP-16 in malignant brain tumors," Journal of Neuro-Oncology, vol. 4, no. 4, pp. 397-401, 1987.

[50] F. H. Hochberg, L. M. Parker, T. Takvorian, G. P. Canellos, and N. T. Zervas, "High-dose BCNU with autologous bone marrow rescue for recurrent glioblastoma multiforme," Journal of Neurosurgery, vol. 54, no. 4, pp. 455-460, 1981.

[51] G. L. Phillips, J. W. Fay, G. P. Herzig et al., "Intensive 1,3-bis(2-chloroethyl)-1-nitrosourea (BCNU), NSC 4366650 and cryopreserved autologous marrow transplantation for refractory cancer. A phase I-II study," Cancer, vol. 52, no. 10, pp. 1792-1802, 1983.

[52] H. Brem, S. Piantadosi, P. Burger et al., "Intraoperative chemotherapy using biodegradable polymers in a prospective, multi-institutional placebo-controlled clinical trial for safety and effectiveness," Neurosurgery, vol. 35, article 574, 1994.

[53] G. Bouvier, R. D. Penn, J. S. Kroin, R. A. Beique, M. J. Guerard, and J. Lesage, "Stereotactic administration of intratumoral chronic chemotherapy of recurrent malignant Gliomas," Applied Neurophysiology, vol. 50, no. 1-6, pp. 223226, 1987.

[54] D. J. Stewart, "Hyperosmolar disruption of the blood-brain barrier as a chemotherapy potentiator in the treatment of brain tumours," in The Gliomas, M. S. Berger and C. B. Wilson, Eds., pp. 570-578, WB Saunders, Philadelphia, Pa, USA, 1999.

[55] C. A. Meyers and P. D. Brown, "Role and relevance of neurocognitive assessment in clinical trials of patients with CNS tumors," Journal of Clinical Oncology, vol. 24, no. 8, pp. 1305-1309, 2006.

[56] M. A. Weitzner, "Psychosocial and neuropsychiatric aspects of patients with primary brain tumors," Cancer Investigation, vol. 17, no. 4, pp. 285-291, 1999.

[57] D. J. Brat, R. A. Prayson, T. C. Ryken, and J. J. Olson, "Diagnosis of malignant glioma: role of neuropathology," Journal of Neuro-Oncology, vol. 89, no. 3, pp. 287-311, 2008.

[58] D. J. Brat, R. A. Prayson, T. C. Ryken, and J. J. Olson, "Diagnosis of malignant glioma: role of neuropathology," Journal of Neuro-Oncology, vol. 89, pp. 287-311, 2008.

[59] L. C. Blake and K. R. Maravilla, "Computed tomography," in The Gliomas, M. S. Berger and C. B. Wilson, Eds., pp. 242-274, WB Saunders, Philadelphia, Pa, USA, 1999.

[60] M. J. Fulham and G. D. Chiro, "Positron emission tomography and ' $\mathrm{H}$-spectroscopic imaging," in The Gliomas, M. S. Berger and C. B. Wilson, Eds., pp. 295-317, WB Saunders, Philadelphia, Pa, USA, 1999.

[61] R. Spiegelmann and W. Friedman, "Closed biopsy techniques," in The Gliomas, M. S. Berger and C. B. Wilson, Eds., pp. 376390, WB Saunders, Philadelphia, Pa, USA, 1999.

[62] D. C. Shrieve, P. H. Gutin, and D. A. Larson, "Central nervous system toxic effects of radiotherapy," in The Gliomas, M. S. Berger and C. B. Wilson, Eds., pp. 737-740, WB Saunders, Philadelphia, Pa, USA, 1999.

[63] J. C. Buckner, "Systemic sequelae of chemotherapy for Gliomas," in The Gliomas, M. S. Berger and C. B. Wilson, Eds., pp. 759-767, WB Saunders, Philadelphia, Pa, USA, 1999. 


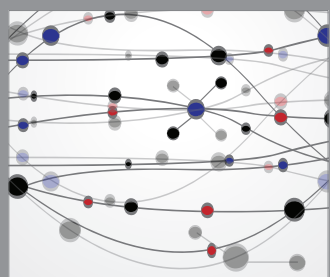

The Scientific World Journal
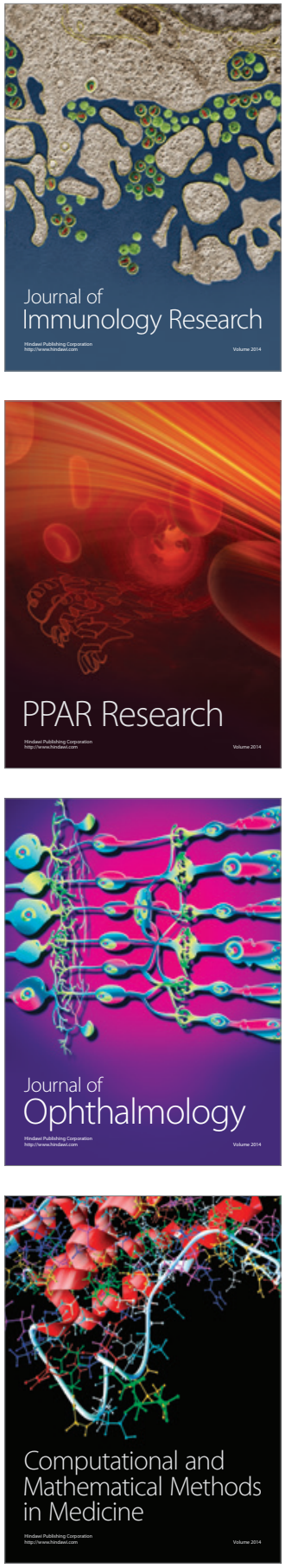

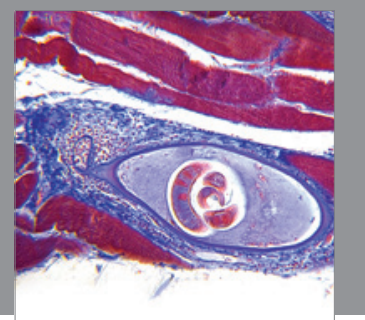

Gastroenterology

Research and Practice
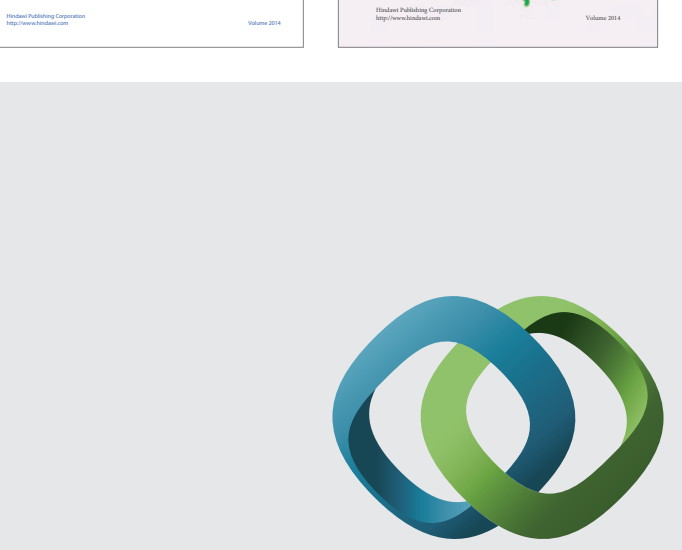

\section{Hindawi}

Submit your manuscripts at

http://www.hindawi.com
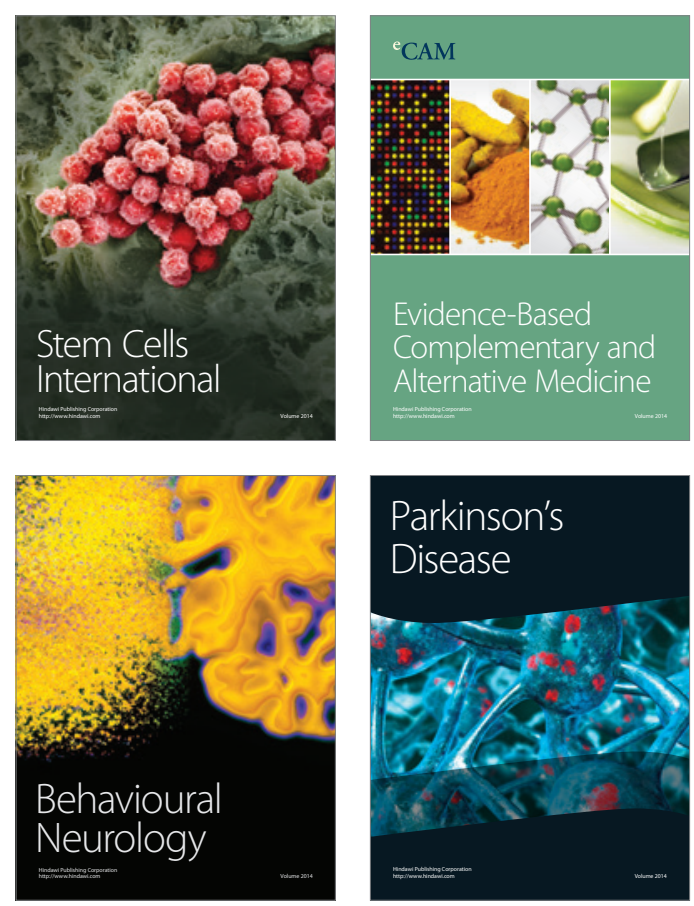

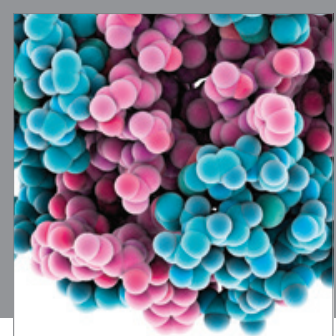

Journal of
Diabetes Research

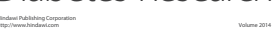

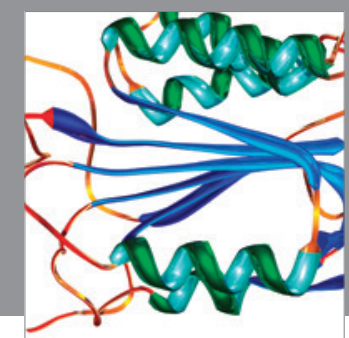

Disease Markers
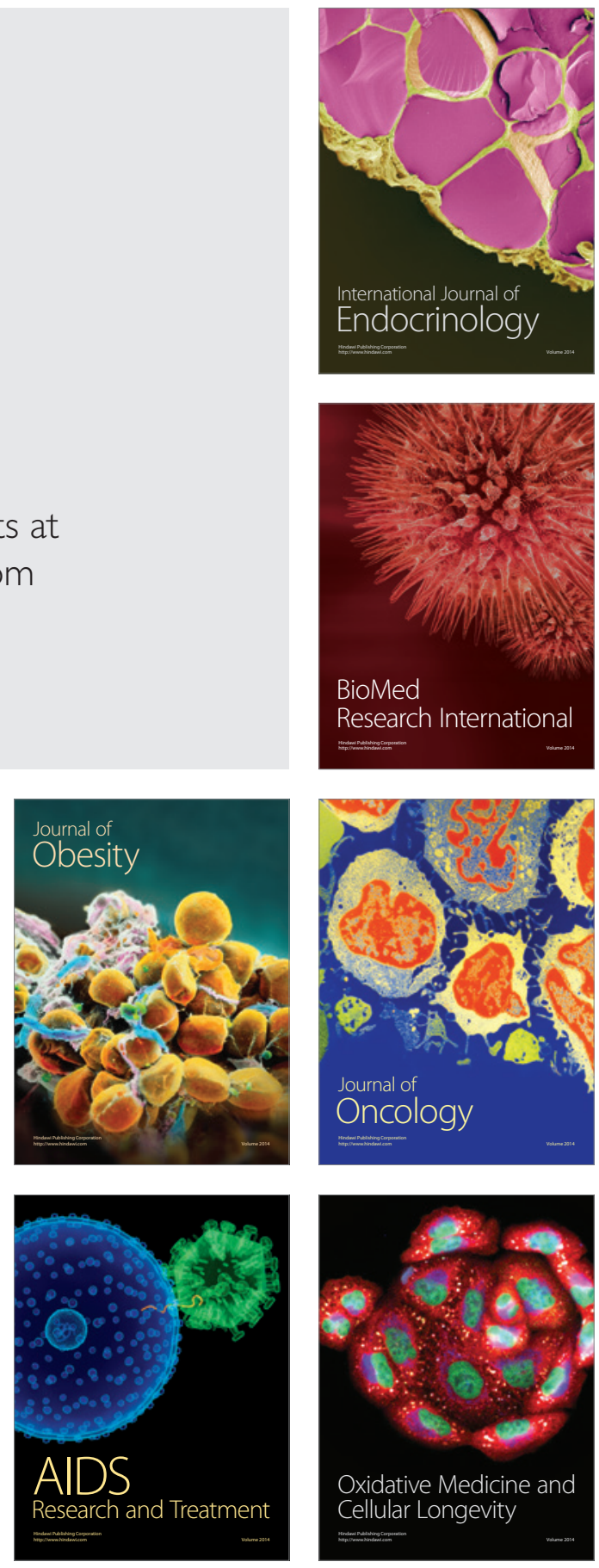